assumptions is not accompanied by any reduction in residual degrees of freedom. $\mathrm{He}$ is thus proceeding as if any set of environmental correlations is equally appealing. ception of the "game" is revealed. However much the assumptions of the Hawaii model might be questioned on biological or psychological grounds, they are nevertheless founded upon a strong yet simple theory of genetic and social transmission. Such a theory comes close to predicting the observed distribution of individual differences in IQ. Science only degenerates into a "game" when additional components are imported post hoc with no clear basis in a theory of gene action or social interaction.

The provision of competing environmental theories which could match the explanatory power is an exciting and challenging prospect. The book devotes three pages to a number of positive suggestions, of which the need to develop
It is here that the author's basic misconclassical genetic theory for simplicity and

environmental theories is but one. It is suggested that IQ should be treated as an index of an underlying construct rather than the actual phenotype of interest; that models for multiple cognitive measures be developed; that models and designs should be explored to allow separation of the developmental recursion of genetic and environmental factors.

Had these suggestions been published at the start of the past decade they would have been heralded as a positive and imaginative contribution. Now, however, Dr Taylor is not alone in his concern for truth. A substantial body of theoretical and empirical research in the wider area of human and behavioural genetics has been published in journals and conference proceedings. Over the past five years a significant start has been made on the study of precisely these issues and in response to those criticisms that the book develops in such detail.

Lindon J. Eaves is Fellow and Tutor in Experimental Psychology at Lady Margaret Hall and Lecturer in Experimental Psychology at the University of $O x$ ford.

\section{How microorganisms get their nitrogen}

\section{Ben Miflin}

\section{Microorganisms and Nitrogen Sources: Transport and Utilization of Amino Acids, Peptides, Proteins, and Related Substrates. Edited by J.W. Payne. Pp.870. (Wiley: 1980.) $£ 45, \$ 126.60$.}

THE lengthy sub-title of this book is intended, according to the editor, to provide a more complete indication of the coverage of the volume. However, even this does not really do justice to the number of topics covered. For example, at least four of the chapters deal with some aspect of nitrogen metabolism in higher plants, one of which is entirely devoted to the subject. Further, although the book set out to cover transmembrane metabolism, it also deals in some detail with the utilization of ammonia, amino acids and peptides. In all there are nearly 900 pages, spread over 26 contributions which are divided into four sections: Amino Acids, Peptides, Proteins, Related Systems. These have been written by a variety of international authors, many prominent in their field. Six chapters cover the transport and utilization of amino acids and peptides in bacteria, yeast and fungi. The genetics, regulation and energetics of the uptake of these nitrogen sources are dealt with separately in a further six chapters. Reflecting our greater knowledge of bacterial biochemistry, relative to that of other organisms, there is discussion of the membrane proteins responsible for amino acid transport. The section on proteins, although still dealing with transport, is more heavily biased towards utilization and re-utilization.
The final section of the book is to me what makes it really worthwhile; it is unusual in that the editor has included a number of contributions that should encourage the readers to broaden their horizons. Thus, there is a chapter on the $\gamma$-glutamyl cycle in animals and speculation as to how it might apply to microorganisms, and two on the utilization of nitrogenous compounds by green autotrophic plants. The utilization of ammonia and amides is well covered in three contributions and the reader is finally informed of some of the other functions of peptides, notably as antimicrobial agents and ionophores.

The contributions have generally been written with the aim of making them a readable and developmental account of the field rather than a combination of the author's latest results and his reference card library which, alas, all too often occurs in multi-authored volumes. The coverage of the literature is generally broad although not exhaustive (or exhausting) and, considering the size and number of authors, is reasonably up to date.

Whilst it is possible to argue with various points expressed and to pick out certain mistakes, this would be ungenerous to an editor and authors who have obviously striven hard to provide an informative and stimulating book, which I am sure will be a standard work in the field for many years to come.

Ben Miflin is Head of the Biochemistry Department at Rothamsted Experimental Station, Harpenden, Hertfordshire.
Complexity of melting

\author{
G. Gee
}

Crystal Melting. Macromolecular Physics, Vol.3. By Bernhard Wunderlich. Pp.363. (Academic: 1980.) \$42.50, £23.80.

THIS volume, the third in the author's treatise Macromolecular Physics, adds three chapters to the seven already published (in 1973 and 1976). The three volumes deal successively with the description of macromolecular crystals, with their growth and now with their melting. Although the author states that the first part of the project is now complete the next volume is evidently planned in some detail, for there are numerous references forward to it for thermodynamic discussions which are needed in this volume. This spreading of intimately interconnected topics over four volumes and ten years is a necessary consequence of a single author undertaking such a huge task, but it is not very helpful to today's reader to be told (p.104): "the derivation is obvious by comparison with the results listed in 11.2.3" (Vol.4).

In any elementary thermodynamics course melting is presented as a simple example of equilibrium between two bulk phases: a perfect crystal and its melt. Why then a whole volume on melting? In the first place, polymer crystals are characteristically small and imperfect: 100 per cent crystallinity is seldom attainable. Even the "melt" may require further specification, for example molecular weight and perhaps cross-linking or (in copolymers) composition. Finally, equilibrium between the two is in most cases almost impossible to achieve. These problems are recognized in the chapter divisions: equilibrium melting; irreversible melting; copolymer and isomer melting. In each chapter a discussion of general principles is followed by a detailed and critical survey of what is considered to be the most important and reliable experimental work.

This volume, like its predecessors, is addressed primarily to the specialist reader. He can safely be assumed to have at least a general knowledge of the topics discussed in other volumes (including those yet to appear). For him it is a splendid book, a reliable guide to the literature, offering a considered judgement but quick to point out difficulties. It is altogether too detailed for a reader wanting only a general account of this aspect of polymer physics. Between the two extremes will be many, engaged perhaps in the making or application of polymers, who will find it a useful source of reference and comment on the behaviour of a particular material.

G. Gee is Emeritus Professor of Chemistry at the University of Manchester.

The second edition of Gregory Bateson's Naven, the classic study of the culture of the Iatmul people of New Guinea, has been reissued by Wildwood House, price $£ 4.50$. 